\title{
Monocular Image Parameter-based Aircraft Sense and Avoid*
}

\author{
Peter Bauer ${ }^{1}$, Balint Vanek ${ }^{1}$, Tamas Peni ${ }^{1}$, Anna Futaki ${ }^{2}$, Borbala Pencz ${ }^{1}$, Akos Zarandy ${ }^{1}$ and Jozsef Bokor ${ }^{1,3}$
}

\begin{abstract}
This paper deals with the problem of monocular image parameter-based sense and avoid. It considers image parameters as decision variables and selects decision thresholds related to collision and non-collision scenarios. The main contribution is non-heuristic threshold selection. Another contribution is the characterization of possible intruder threats with sizespeed curves given in closed form formulae. This makes it possible to avoid the use of lookup tables. The overall decision and avoidance concept is evaluated in a software-in-the-loop simulation campaign considering threats ranging from small UAV to large airliner. The miss detection rate of the method is zero which is an excellent result, however the false alarm rate is high. The causes of this are pointed out and targeted as further developments. Finally, promising results are presented executing the method for real camera images.
\end{abstract}

Index Terms-Sense and avoid, Monocular camera, Image parameter, Threshold selection, Test campaign

\section{INTRODUCTION}

Sense and avoid (S\&A) capability is a crucial ability for the future unmanned aerial vehicles (UAVs). It is vital to integrate civilian and governmental UAVs into the common airspace according to [1] and [2]. At the highest level of integration (called Dynamic Operation in [2]) Airborne Sense and Avoid (ABSAA) systems are required to guarantee airspace safety.

In this field the most critical question is the case of noncooperative S\&A for which usually complicated multi-sensor systems are developed (see [3] for example). However, in case of small UAVs the size, weight and power consumption of the onboard S\&A system should be minimal. Monocular vision based solutions can be cost and weight effective therefore especially good for small UAVs [4], [5], [6], [7]. These systems basically measure the position (bearing) and size of intruder aircraft $(\mathrm{A} / \mathrm{C})$ camera image without range information. [5] introduces a collision detection method based only on intruder bearing and size. However, the method is implemented with heuristic threshold selection through Monte Carlo simulations (10.000 cases) and repeated avoidance maneuvers can occur for the same threat because of non proper thresholds.

The current article targets to derive a similar method with non-heuristic threshold selection using the possible minimum number of system parameters and avoiding time consuming

\footnotetext{
*This work is supported by the Office of Naval Research Global, Grant Number N62909-10-1-7081, Dr. Charles Holland program officer.

${ }^{1}$ Author is with Institute for Computer Science and Control, Hungarian Academy of Sciences (MTA SZTAKI), Budapest, Hungary Corresponding author: peter.baueresztaki.mta.hu

${ }^{2}$ MSc student at Faculty of Transportation Engineering and Vehicle Engineering, Budapest University of Technology and Economics

${ }^{3}$ Author is with MTA-BME Control Engineering Research Group
}

simulations for tuning. The theoretical results are applied to select appropriate thresholds and a software-in-the-loop (SIL) simulation campaign is conducted to evaluate performance. Additionally an avoidance maneuvering strategy is proposed and implemented. Finally, first experiences with real camera images are evaluated.

The article is divided into eight sections. Section II summarizes the derived methodology for threshold selection. Section III characterizes considered own craft categories, airspace segments and intruder categories accordingly together with S\&A system effectiveness requirements. Section IV introduces the SIL test environment. Then section V selects the detection thresholds and section VI summarizes test results. VII evaluates the first camera experiences. Finally section VIII concludes the paper.

\section{Summary OF PROPOSED METHODOLOGY}

The applied basic notations (image parameters) are shown in Fig. 1.

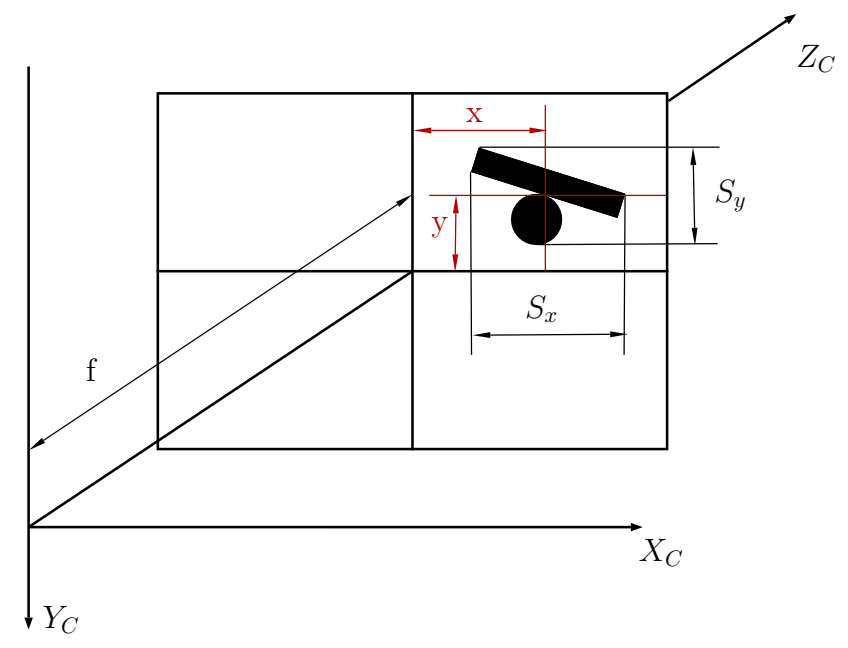

Fig. 1. Considered image parameters

In $X_{C}, Y_{C}, Z_{C}$ camera frame $x, y$ are the positions of intruder image centroid (IIC) and $S_{x}, S_{y}$ are the intruder image sizes (IIS) (horizontal / vertical). A pinhole camera model is used which relates image parameters $\left(x, y, S_{x}, S_{y}\right)$ to own aircraft camera focal length $f$, intruder position $\left(X, Y, Z\right.$ ) in camera frame, intruder size $R_{x / y}$ (horizontal / vertical), intruder relative velocities $V_{x}, V_{y}, V_{z}$ in camera frame, time to collision $t_{T C}$ (defined to go to zero as the aircrafts approach each other), miss distances at $\mathrm{Z}=0 X_{a}, Y_{a}$ and relative miss distances $C P A=X_{a} / R_{x}$ or $Y_{a} / R_{y}$ (called closest point of approach CPA). The basic equations of pinhole camera model are: 


$$
\begin{aligned}
& x=f \frac{X}{Z}, \quad y=f \frac{Y}{Z} \\
& S_{x}=f \frac{R_{x}}{Z}, \quad S_{y}=f \frac{R_{y}}{Z}
\end{aligned}
$$

Considering the intruder relative velocities and $t_{T C}$ the above expressions can be reformulated and their derivatives derived. From now, formulae are presented only for the $x$ horizontal direction because the $y$ direction formulae are structurally the same.

$$
\begin{aligned}
& x=-f\left(\frac{R_{x}}{V_{z}} \frac{C P A}{t_{T C}}-\frac{V_{x}}{V_{z}}\right), \quad S_{x}=-f \underbrace{\frac{R_{x}}{V_{z}}}_{R_{V}} \frac{1}{t_{T C}} \\
& \frac{d x}{d t}=-\frac{f}{V_{z}} \frac{X_{a}}{t_{T C}^{2}}=-f R_{V} \frac{C P A}{t_{T C}^{2}}, \quad \frac{d S_{x}}{d t}=-f R_{V} \frac{1}{t_{T C}^{2}}
\end{aligned}
$$

In a S\&A scenario the miss distance $X_{a}$ should be estimated. This would be possible from $(d x / d t) /\left(d S_{x} / d t\right)$ if the intruder size $R_{x}$ were known. However, it is unknown. Inspecting closer the ratio (derived also in [8]):

$$
\frac{d x / d t}{d S_{x} / d t}=\frac{X_{a}}{R_{x}}=C P A
$$

CPA well characterizes the miss distance relative to intruder size. This is a perfect parameter for collision estimation. If $C P A=0$ mid-air collision (MAC) is sure. If $0<C P A<$ $C P A_{c}$ (below a selected threshold) near mid-air collision (NMAC) can be defined. $d x / d t$ and $d S_{x} / d t$ can only be estimated from measured $x$ and $S$ (from now $S_{x}$ will be denoted as $S$ and $R_{x}$ as $R$ for simplicity) and so they can be noisy and this could lead to false $C P A$ estimates. On the other hand $|d x / d t| \gg 0$ is good indicator of non-collision and $|d S / d t| \rightarrow \infty$ of collision. So, detection thresholds $\dot{x}_{L I M}$ and $\dot{S}_{L I M}$ should be selected for them. The proposed threshold selection methodology is summarized below.

1) Decide about the collision decision time $t_{T C}=t_{C d e c}$ required to be able to execute the avoidance maneuver in safe distance from the intruder. This depends on own craft dynamics and intruder velocity.

2) Determine $\dot{S}_{L I M}$ to decide about collision based-on $d S / d t$

3) Determine $t_{S d e l}$ and $t_{x d e l}$ decision delays because of camera image pixelization and sampling ('noises').

4) Decide about the $C P A_{C}$ limit, below which all the scenarios should be decided to be NMAC (including MACs also).

5) Determine $\dot{x}_{L I M}$ based on $C P A_{C}$ and the derived formulae.

6) Determine $C P A_{N C}$ limit above which all the scenarios are determined to be non-collision.

The next section characterizes own craft and possible intruder threats so setting the parameter space for tuning of methodology. The steps will be explained in detail in section V.

\section{ChaRACTERIZATION OF POSSIBLE SCENARIOS}

In this development the own craft is considered as the Aerosonde UAV which model was constructed based on [9]. Its cruise speed ranges between 17 and $23 \mathrm{~m} / \mathrm{s}$ obtained from the simulation of the model.

The possible airspace categories are selected based on [10] which makes an important effort to set S\&A system effectiveness standards considering different class of UAVs and airspaces. The targeted airspaces by current development are Class $\mathrm{D} / \mathrm{E}$ and $\mathrm{G}$ which does not require on-board transponder or ATC link. The targeted own craft categories are Group 1 to 4 (micro to tactical). The overall S\&A system effectiveness requirement laid down in [10] ranges from $30.9 \%$ to $68.9 \%$ for these categories. So, this target should be satisfied by the proposed algorithm if possible.

Considering the possible threats, in Class D/E airspaces the intruder aircrafts can range from micro UAVs through general aviation (GA) aircraft until large airliners / transporters on their approach to airports. So, these types should be characterized. [10] characterizes $\mathrm{A} / \mathrm{Cs}$ based-on their weight and speed, however from a vision sensor point of view it is better to use size and speed. Wingspan $(b)$, fuselage length $(L)$ and cruise speed characteristics were collected from [11] ranging from CAP-10 to Airbus A380 and AN225 including also helicopters. At first, the relation between wingspan and fuselage length was examined (rotor diameter and fuselage length). It is almost linear, so an average size $((b+L) / 2)$ can well characterize the $\mathrm{A} / \mathrm{Cs}$ (from now including helicopters also) and is used in the sequel. At the next step the size-speed characteristic was plotted as shown in Fig. 2. A least squares optimal size-speed curve was fitted on the data and the minimum and maximum deviations were characterized by simple functions generating boundary curves for the plotted points applying trial and error. From this, the speed range of a given size of $\mathrm{A} / \mathrm{C}$ can be calculated in closed form. Below $\mathrm{R}=7 \mathrm{~m}$ size, only UAVs are assumed with $\mathrm{min} . / \mathrm{max}$. speeds as $10 \mathrm{~m} / \mathrm{s}$ and $40 \mathrm{~m} / \mathrm{s}$ respectively.

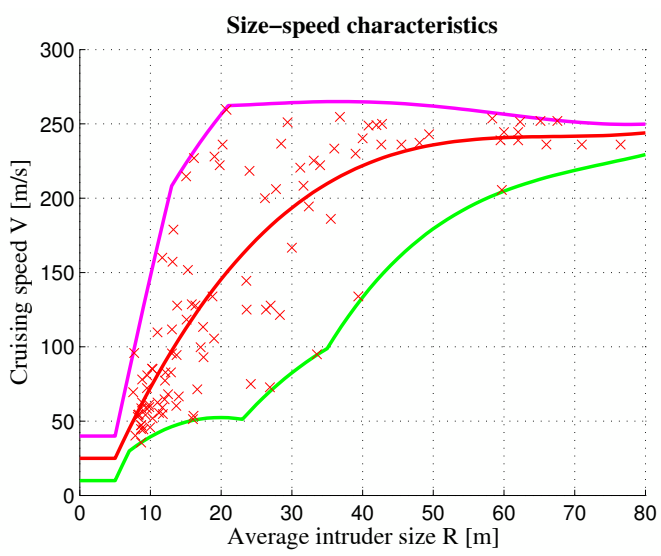

Fig. 2. Aircraft size-cruising speed characteristics. The crosses represent original data

The average speed from $\mathrm{A} / \mathrm{C}$ average size (middle curve in Fig. 2): 


$$
V_{i 0}=8.75 \cdot 10^{-4} R^{3}-0.177 \cdot R^{2}+12 \cdot R-30.7
$$

The positive maximum deviation from $V_{i 0}$ (upper bound):

$$
\begin{aligned}
& \Delta V^{+}=15 \text { if } R \leq 5 \\
& \Delta V^{+}=15+12(R-5) \quad \text { if } \quad 5<R \leq 13 \\
& \Delta V^{+}=111 \text { if } 13<R<21 \\
& \Delta V^{+}=111 e^{-(R-21) 0.05} \quad \text { if } \quad 21 \leq R
\end{aligned}
$$

The negative maximum deviation from $V_{i 0}$ (lower bound):

$$
\begin{aligned}
& \Delta V^{-}=-15 \text { if } R \leq 7 \\
& \Delta V^{-}=-15-6(R-7) \text { if } 7<R \leq 23 \\
& \Delta V^{-}=-111 \text { if } 23<R<35 \\
& \Delta V^{-}=-111 e^{-(R-35) 0.045} \text { if } 35 \leq R
\end{aligned}
$$

This way the intruder velocity ranges can be characterized depending on the size of intruder. However, the $R_{V}$ term in (2) strongly depends on the direction of intruder relative to own craft because $V_{z}=V_{o}+V_{i} \cos (\beta) \cos (\alpha)$ is only a component of relative speed. Here $\alpha$ and $\beta$ characterizes direction of the intruder as shown in Fig. 3.

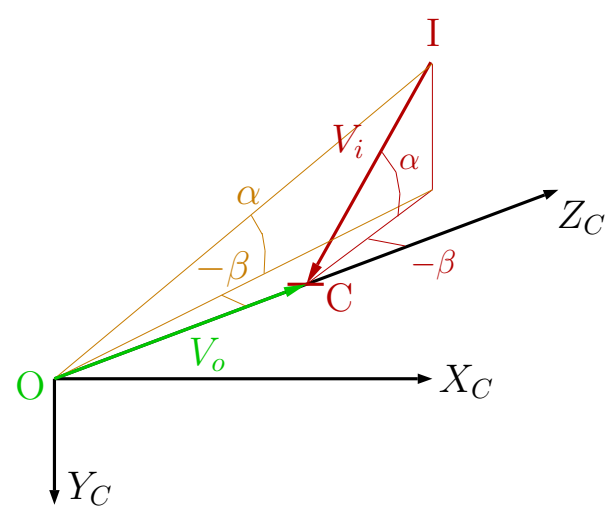

Fig. 3. Angles in collision scenario

Consequently $R_{V}$ can be characterized as follows:

$$
R_{V}=\underbrace{\frac{R}{V_{o}+V_{i} \cos (\beta) \cos (\alpha)}}_{V_{z}}=k \underbrace{\left(\frac{R}{V_{o}+V_{i}}\right)}_{R_{V 0}}
$$

However, $\beta$ and $\alpha$ can not be measured from image parameters, $\beta^{\prime}$ and $\alpha^{\prime}$ are measured instead (see formula with $f, x, y$ below). Later a numerically approximated lower bound $k_{b}$ will be applied instead of $k$ :

$$
\begin{aligned}
& \cos \left(\beta^{\prime}\right) \cos \left(\alpha^{\prime}\right)=\frac{f}{\sqrt{f^{2}+x^{2}}} \frac{f}{\sqrt{f^{2}+y^{2}}} \\
& k_{b}=\frac{1}{\left(\cos \left(\beta^{\prime}\right) \cos \left(\alpha^{\prime}\right)\right)^{0.8}} \leq k
\end{aligned}
$$

After summarizing own craft and intruder characteristics the SIL simulation environment used in the test campaign is briefly introduced.

\section{SIL SIMULATION ENVIRONMENT}

A simulation environment is built in Matlab Simulink to generate collision and non-collision scenarios and apply the proposed methodology together with avoidance maneuvers.

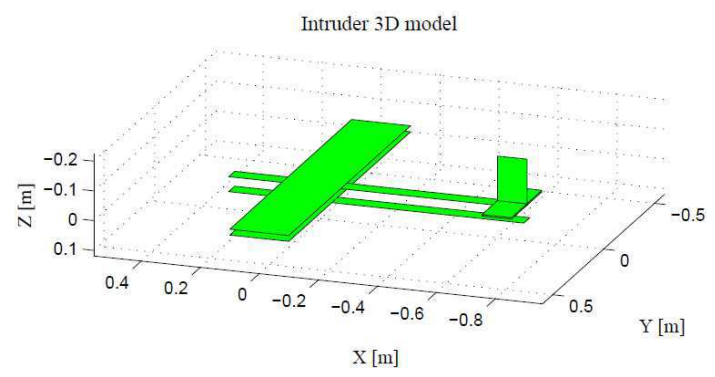

Fig. 4. Intruder 3D model (only the vertices are considered)

In the simulated scenarios the following assumptions and methods are applied:

- Intruder is in front of own craft (inside $\pm 75^{\circ}$ horizontal $(\alpha), \pm 30^{\circ}$ vertical $(\beta)$ field of view)

- Both own craft and intruder fly straight paths with constant velocity before avoidance starts.

- Only one intruder threatens until the conflict is solved.

- The intruder is non-cooperative and is not equipped with S\&A system

- A fixed onboard monocular camera is applied with pixelization errors and sampling $(\Delta t=0.07 \mathrm{~s}$ means about 14-15 fps of the real camera [12]) in intruder image centroid (IIC) and size (IIS).

- The intruder can range from small UAV to large transport or airliner.

- The own craft attitude is known without errors.

- Own craft motion is completely simulated with autopilot performing waypoint tracking and avoidance if required.

- A pinhole camera model is applied considering the transformations between earth, body and camera frame. A 3D vertex set is applied to model the intruder (see Fig. 4) which is scalable to different sizes. $S_{x}$ and $S_{y}$ are obtained as maximum horizontal / vertical sizes of the vertex set in image plane (see Fig. 1). Another assumption is the unlimited field of view of camera.

- Ego motion of own craft was compensated in $(x, y)$ centroid position but was not compensated in intruder size $\left(S_{x}, S_{y}\right)$

- The intruder motion is simulated without orientation and velocity changes because otherwise the miss distance can become different because of intruder dynamics.

- Intruder directions are considered in the ranges $\beta=$ $-75: 25: 75^{\circ}, \alpha=-30: 15: 30^{\circ}$ and the miss distances are defined as $D_{a}=R_{0} \cdot s c l \cdot C P A\left(R_{0}=\right.$ $1.2 \mathrm{~m}$ ) in given directions (left/right up/down) from own craft characterized by $\tau$ as shown in Fig. 5. scl is the scale from $R_{0}$ intruder size to $R$.

Of course real situations can violate some of these assumptions the examination of these cases should be the topic of future work. 


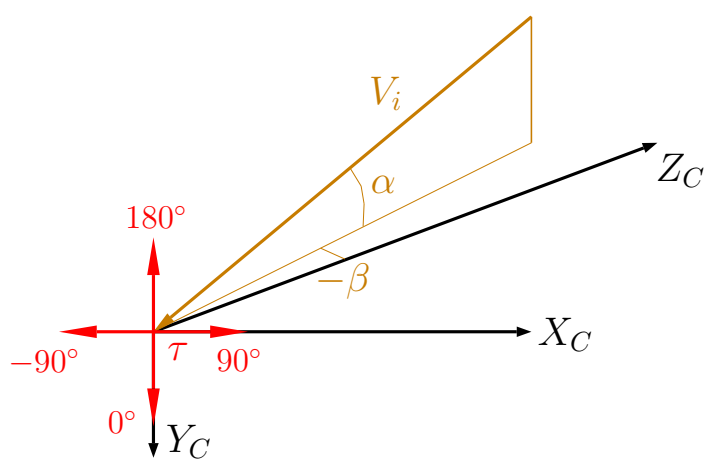

Fig. 5. Intruder flight directions (in own craft camera system)

\section{FORMULAE BASED THRESHOLD SELECTION}

Assume first that $\dot{S}_{L I M}$ and $\dot{x}_{L I M}$ are selected. Then the collision (C) and non-collision (NC) decision times can be calculated from the continuous functions (2):

$$
\begin{aligned}
& t_{C d e c}=\sqrt{-f \cdot k R_{V 0} / \dot{S}_{L I M}} \\
& t_{N C d e c}=\sqrt{-f \cdot k R_{V 0} C P A / \dot{x}_{L I M}}
\end{aligned}
$$

However, pixelization and sampling will cause delays in the violation of thresholds $\dot{S}_{L I M}$ and $\dot{x}_{L I M}$ and this should be considered in their selection. The scheme for this can be seen in Fig. 6.

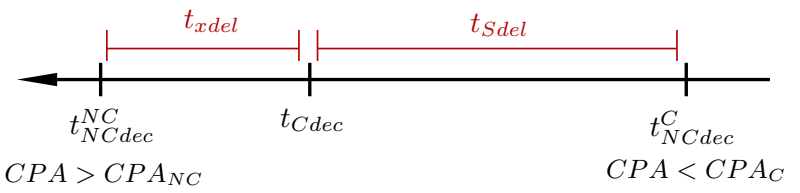

Fig. 6. Overall scheme of threshold selection

Here, $t_{C d e c}$ is the time of collision decision (time $\left(t_{T C}\right)$ when it is decided that $\mathrm{A} / \mathrm{Cs}$ are on collision path) and $t_{S d e l}$ is its maximum delay. $t_{N C d e c}^{C}$ is the time of false noncollision decision if aircrafts are in MAC / NMAC scenario. It should never be before $t_{C d e c}$ to guarantee $100 \%$ collision detection. $t_{N C \text { dec }}^{N C}$ is the time of non-collision decision if aircrafts are out of NMAC range. It should never be after $t_{C d e c}$ (that means false collision decision), its worst case delay is $t_{x d e l}$. Of course there will be a hysteresis between guaranteed collision $\left(C P A<C P A_{C}\right)$ and non-collision $\left(C P A>C P A_{N C}>C P A_{C}\right)$ decisions by this scheme (note that time to collision decreases from left to right in the figure). The proposed threshold selection methodology (see section II) is implemented step by step in the sequel.

To select the required collision decision time $\left(t_{C d e c}\right)$ the avoidance strategy should be determined. [5] proposes to turn towards the intruder if collision is detected. This is a suitable strategy for non-cooperative intruders w/o S\&A equipment. In this work it is completed with a return strategy to original path. In case of collision detection the own craft turns towards intruder until it is at $+80^{\circ}$ or $-80^{\circ}$ in horizontal field of view. Then it tracks the intruder with this $+/-80^{\circ}$. If own flight direction is again towards the original path the controller switches to track the path again.
$t_{C d e c}$ can be selected by simulating (measuring for real $\mathrm{A} / \mathrm{C}$ ) the turning maneuver of own craft (time required to $90^{\circ}$ turn and distance from collision point at end of turn) and considering possible intruder speeds and required minimum distance. The level turns of Aerosonde were simulated for 17, 20 and $23 \mathrm{~m} / \mathrm{s}$ in the SIL environment. Fig. 7 shows the turning paths together with the original straight path and possible collision points (C) as intersections of straight path and horizontal lines for the different velocities.

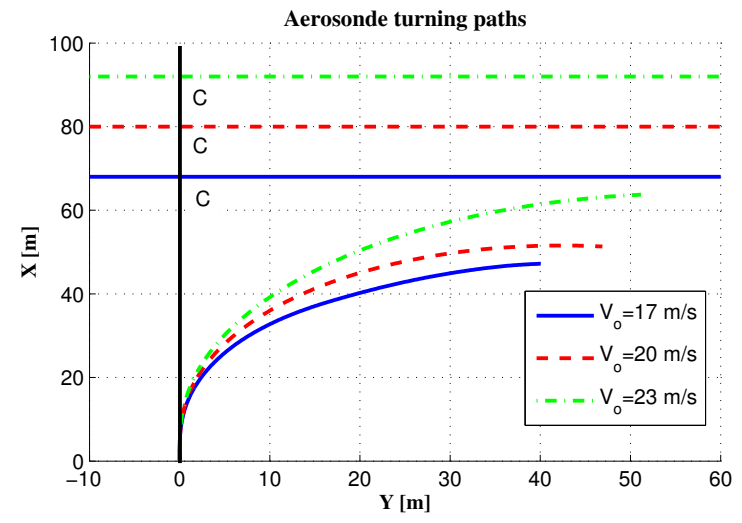

Fig. 7. Turning paths of Aerosonde

4 seconds were enough for $90^{\circ}$ turn in all cases and the collision points are about $20 \mathrm{~m}$ away from turned $\mathrm{A} / \mathrm{C}$ in $\mathrm{X}$ direction. Simulation calculations were made with $R=0.5: 0.5: 80 \mathrm{~m}$ intruder sizes and the corresponding minimum, middle and maximum intruder speeds from Fig. 2 considering $\beta=0: 75^{\circ}$ and all three own craft trajectories from Fig. 7. The intruder was simulated to fly to the collision point without avoidance with its actual velocity and $\beta$ direction starting from time $4 \mathrm{~s}$ before collision. The distance between own craft and intruder trajectories was calculated at every $0.01 \mathrm{~s}$. The absolute min. distance resulted as $38 \mathrm{~m}$ for small size intruders, so the avoidance was estimated to be acceptable in every case.

Thus decision at $t_{C d e c}>4 \mathrm{~s}$ guarantees that collision can be avoided. However, the horizontal limit distance for NMAC is given as about $150 \mathrm{~m}$ for GA A/C in [13]. So, this minimum distance should be guaranteed in avoidance $(38 \mathrm{~m}$ is not satisfactory) (GA wingspan is about $10 \mathrm{~m}$, so $150 \mathrm{~m}$ is about $\mathrm{CPA}=15$ ). In case of small UAV intruders $20 \mathrm{~m}$ is decided to be guaranteed as minimum (with $1 \mathrm{~m}$ wingspan this is $\mathrm{CPA}=20$ ). To increase safety it is assumed that own craft is in line with the collision point after $4 \mathrm{~s}$ and $90^{\circ}$ turn. This means $0 \mathrm{~m} \mathrm{X}$ distance from the $\mathrm{C}$ points in Fig. 7. Thus $t_{C d e c}>4 s$ should be applied to guarantee limit distances. Own craft maximum speed is $23 \mathrm{~m} / \mathrm{s}$. For UAV intruder $R=1 \mathrm{~m}$ the maximum speed is $V_{i}=40 \mathrm{~m} / \mathrm{s}$. To have guaranteed $20 \mathrm{~m}$ minimum distance after $4 \mathrm{~s}$ turn $t_{C d e c}$ should be increased by $20 / 63 \approx 0.3 \mathrm{~s}$ to $t_{C d e c}=4.3 \mathrm{~s}$. For GA intruder $R=7.5 \mathrm{~m}$ is the minimum size and $V_{i}=72 \mathrm{~m} / \mathrm{s}$ is the maximum speed. To have $150 \mathrm{~m}$ minimum distance $t_{C d e c}$ should be increased by $150 / 95 \approx 1.6 \mathrm{~s}$ to $t_{C d e c}=5.6 \mathrm{~s}$.

Examining $t_{C d e c}$ in (8) shows that the $\dot{S}_{L I M 0}$ threshold should be determined with minimum $k(\min (k)=$ 
$\left.\min \left(k_{b}\right)=1\right)$ and $\min \left(R_{V 0}\right)$ because increase in either $k$ or $R_{V 0}$ will increase $t_{C d e c}$ for a given $\dot{S}_{L I M 0}$ and so makes an earlier collision decision. Minimum $R_{V 0}$ means the minimum of $\frac{R}{V_{o}+V_{i}}$. Considering the given maximum speeds

$$
\dot{S}_{L I M 0}=f \cdot \min \left(R_{V 0}\right) \frac{1}{t_{C d e c}^{2}}=0.51
$$

results for UAV and $\dot{S}_{L I M 0}=1.51$ for GA intruder. Selecting the smaller one and considering the effect of $k$ as $k_{b}$ gives finally:

$$
\dot{S}_{L I M}=k_{b} \cdot \dot{S}_{L I M 0}=k_{b} \cdot 0.51
$$

The decision criterion to determine $\dot{x}_{L I M}$ is (here $t_{N C d e c}^{C}$ is the decision time of non-collision based on $d x / d t$ threshold violation):

$$
t_{\text {Cdec }}-t_{\text {Sdel }}>t_{N C d e c}^{C}
$$

where

$$
\begin{aligned}
& t_{N C d e c}^{C}=\sqrt{-f k R_{V 0} C P A_{C} / \dot{x}_{L I M}} \\
& t_{S d e l} \leq(\max (r)+1) \Delta T \\
& r=\frac{-t_{2}+\sqrt{t_{2}^{2}+t_{2} \Delta T+\frac{\Delta t}{\Delta T} t_{2}^{2}+t_{2} \Delta t}}{\Delta T} \\
& \Delta T=\frac{t_{C d e c}^{2}}{-f k R_{V 0}+t_{C d e c}} \\
& t_{2}=t_{C d e c}+\Delta T
\end{aligned}
$$

The above formulae can be derived considering pixelization and sampling of the $d S / d t$ curve but the details are omitted because of space constraints. $\dot{x}_{L I M}$ can be calculated considering worst case values for $k$ and $R_{V 0}$. First $\max (r)$ is determined for $t_{C d e c}$ with $k=k_{b}$ because $t_{N C d e c}^{C}$ moves together with $t_{C d e c}$ if $k$ changes (their ratio is always the same see (8)). So an $R_{V 0}$ sweep is done considering $R=0.5: 0.5: 80 \mathrm{~m}$ and adding the minimum and maximum velocities from Fig. 2 to the minimum $(17 \mathrm{~m} / \mathrm{s})$ and maximum $(23 \mathrm{~m} / \mathrm{s})$ own velocities. This way four curves are obtained for possible $r$ values and plotted against $\mathrm{R}$. The worst case is the overall maximum which resulted to be $\max (r)=0.766$. The criterion for $\dot{x}_{L I M}$ selection finally becomes $\left(\dot{S}_{L I M}=k_{b} \cdot \dot{S}_{L I M 0}\right.$ is substituted into $\left.t_{C d e c}\right)$ :

$$
\begin{aligned}
& \sqrt{-f\left(k / k_{b}\right) R_{V 0} / \dot{S}_{L I M 0}}-1.766 \Delta T> \\
& >\sqrt{-f k R_{V 0} C P A_{C} / \dot{x}_{L I M}} \\
& \Delta T=\frac{-f\left(k / k_{b}\right) R_{V 0} / \dot{S}_{L I M 0}}{-f k R_{V 0}+\sqrt{-f\left(k / k_{b}\right) R_{V 0} / \dot{S}_{L I M 0}}} \\
& \sqrt{\dot{x}_{L I M}}>\sqrt{C P A_{C}} \frac{\sqrt{k f R_{V 0}} k_{b} \dot{S}_{L I M 0}+\sqrt{k_{b} \dot{S}_{L I M 0}}}{\sqrt{k f R_{V 0}} \sqrt{k_{b} \dot{S}_{L I M 0}}-0.766}
\end{aligned}
$$

The coefficient of $\sqrt{C P A_{C}}$ should be maximal to obtain worst case results. However, it is a complicated expression so numerical calculations are required to determine the maximum. A sweep calculation is done for $k\left(k_{b}\right.$ is considered as a parameter) and $R_{V 0}$ considering $R=0.5: 0.5: 80 \mathrm{~m}$, adding the minimum and maximum velocities from Fig. 2 to the minimum $(17 \mathrm{~m} / \mathrm{s})$ and maximum $(23 \mathrm{~m} / \mathrm{s})$ own velocities and considering also $\alpha=-30: 10: 30^{\circ}$ and $\beta=-90: 10: 90^{\circ}$ intruder flight angles (see Fig. 3). These calculations show that the minimum $\sqrt{k f R_{V 0}}$ value gives the maximum for the coefficient of $\sqrt{C P A_{C}}$. This minimum is obtained as $\min \left(\sqrt{k f R_{V 0}}\right)=2.1822$ and this results in the following final expression (substituting also $\left.\dot{S}_{L I M 0}=0.51\right)$ :

$$
\sqrt{\dot{x}_{L I M}}>\sqrt{C P A_{C}} \frac{1.1129 k_{b}+\sqrt{0.51 k_{b}}}{1.5584 \sqrt{k_{b}}-0.766}
$$

$C P A_{C}=10$ was selected as the limit of collision cases (15 and 20 limits were decreased to not to be over conservative), below this the situation should be considered as NMAC. The $k_{b}$ parameter range considering $\alpha=-30: 30^{\circ}$ and $\beta=$ $-75: 75^{\circ}$ and all the possible intruder velocities from Fig. 2 together with the $V_{o}$ range results as $k_{b}=1: 4.064$. This leads to an $\dot{x}_{L I M}$ range of:

$$
\dot{x}_{L I M}=53.18: 63
$$

The next step is to determine $C P A_{N C}$ above which a non-collision decision is guaranteed. This requires to know the worst case time delay in $t_{N C \text { dec }}^{N C}$ called $t_{x d e l} . d x / d t$ is estimated by fitting a quadratic function $x \approx x_{f}=a_{2} s^{2}+$ $a_{1} s+a_{0}$ to the measured $x$ values on a given moving horizon $N$. From now $t$ will denote time to collision which goes to zero, and $s$ will denote the real time which goes to infinity. One to one correspondence is defined between $t$ and $s$ with the same indices. Given an actual time instant $s_{i} x_{f}$ is fitted to data $x\left(s_{i-N-1}\right): x\left(s_{i}\right), \quad N \in \mathbb{Z}$ and $(d x / d t) \mid t_{i} \approx$ $d x_{f} / d t=2 a_{2} s_{i}+a_{1}$.

The time delay for $d x / d t$ can be estimated from the difference between $d x / d t$ (see (2)) and $d x_{f} / d t=2 a_{2} s_{i}+a_{1}$. Fitting the function $x_{f}=a_{2} s^{2}+a_{1} s+a_{0}$ to $x=1 / t$ on a horizon $N$ and comparing $d x_{f} / d t=2 a_{2} s_{i}+a_{1}$ to $d x / d t=1 / t_{i}^{2}$ will characterize the errors. At first, $t_{i}=t_{C d e c}$ should be selected, then $t_{i}$ should be increased by $\Delta t$ steps (shifting the whole $N$ horizon) until $2 a_{2} s_{i}+a_{1}>1 / t_{i}^{2}$ is achieved. The number of required steps $M_{\max }$ gives the time delay $t_{x d e l}=M_{\max } \Delta t$. Doing this calculations around $t_{C d e c}=4 s$ with $N=20$ resulted in $M_{\max }=2$ so $t_{x d e l}=2 \Delta t=0.14 s$.

From the relations

$$
\begin{aligned}
& t_{N C d e c}^{N C}-t_{x d e l}>t_{C d e c} \\
& t_{N C d e c}^{N C}=\sqrt{-f k R_{V 0} C P A_{N C} / \dot{x}_{L I M}}
\end{aligned}
$$

$C P A_{N C}$ can be easily determined.

$$
\begin{aligned}
& \sqrt{-f k R_{V 0} C P A_{N C} / \dot{x}_{L I M}}-0.14> \\
& >\sqrt{-f\left(k / k_{b}\right) R_{V 0} / \dot{S}_{L I M 0}} \\
& \sqrt{\dot{x}_{L I M}}<\sqrt{C P A_{N C}} \frac{\sqrt{f k R_{V 0}} \sqrt{\dot{S}_{L I M}}}{\sqrt{f k R_{V 0}}+0.14 \sqrt{\dot{S}_{L I M}}}
\end{aligned}
$$


Here, the minimum coefficient for $\sqrt{C P A_{N C}}$ should be selected. It is obtained with $\dot{S}_{L I M}=\dot{S}_{L I M 0}$ and $\min \left(\sqrt{k f R_{V 0}}\right)=2.1822$ as:

$$
\sqrt{\dot{x}_{L I M}}<\sqrt{C P A_{N C}} 0.68286
$$

Considering this result and $\dot{x}_{L I M}=53: 63$ leads to $C P A_{N C}=113.7: 135.1$ which seems to be too large. The large $\dot{x}_{L I M}$ values result from the large worst case $t_{S d e l}$. Modifying $t_{S d e l}$ to be: $t_{S d e l}=\Delta T$ (instead $1.766 \Delta T$ ) we get finally from $t_{C d e c}-t_{S d e l}>t_{N C d e c}^{C}$ :

$$
\sqrt{\dot{x}_{L I M}}>\sqrt{C P A_{C}}\left(\sqrt{\dot{S}_{L I M}}+\frac{1}{\sqrt{k f R_{V 0}}}\right)
$$

Again the maximum coefficient is required on the right hand side and this finally leads to:

$$
\sqrt{\dot{x}_{L I M}}>\sqrt{C P A_{C}}\left(\sqrt{0.51 k_{b}}+0.4593 \frac{1}{\sqrt{k_{b}}}\right)
$$

Selecting $C P A_{C}=20$ (to somehow compensate smaller $t_{S d e l}$ and return back to originally required $\mathrm{CPA}=15-20$ ) results in lower bounds $\dot{x}_{L I M}=28: 56$ and $C P A_{N C}=$ 59 : 119. Meanwhile calculating $C P A_{C}$ from the formula with larger $t_{S d e l}(10)$ considering $\dot{x}_{L I M}=28: 56$ gives $C P A_{C}=5.2: 8.8$ so, this modified threshold selection will guarantee collision detection for $C P A_{C}<5$ and noncollision detection for $C P A_{N C}>120$. In the hysteresis part a transient is expected with decreasing number of collision and increasing number of non-collision decisions. The SIL simulation campaign is also conducted to decide about the real $C P A_{C}$ limit if it is $\mathbf{5}$ or above.

\section{TEST RESUlts}

In the simulation campaign the own craft velocity was 20 $\mathrm{m} / \mathrm{s}$, intruder sizes $R=\left[\begin{array}{llllll}1.2 & 5 & 10 & 20 & 40 & 60\end{array}\right] \mathrm{m}$ were considered (from small UAV to large airliner / transport) with $\beta$ and $\alpha$ ranges listed in section IV. $\tau$ was cyclically varied using a counter from $\tau=\left[\begin{array}{llll}0^{\circ} & 90^{\circ} & 180^{\circ} & -90^{\circ}\end{array}\right]$ values. Avoidance maneuver was executed if required. The percent of collision (C) decisions relative to the number of simulated cases is summarized in Table I.

The results show that there is no miss detection until $\mathrm{CPA}=20$ (well above the worst case theoretical value 5) which is the selected non-conservative $C P A_{C}$ limit. For $\mathrm{CPA}=60$ and 120 the number of collision decisions decreases as expected. For CPA=120 theoretically all cases should be non-collision which means that there the percent is the ratio of false alarms (FAs). This is between 0 and $51.4 \%$. The latter is a very large FA rate.

Examining the causes of such a high ratio of FAs resulted in the following two observations:

1) The intruder sizes $S_{x}$ and $S_{y}$ are not ego motion compensated and this results in latent size velocities which could violate the threshold and cause a FA. Removing the ego motion from the simulation (own craft orientation is fixed) decreases the FA rates for $\mathrm{CPA}=120$ between 0 and $28.6 \%$. This is better but also not acceptable.
2) The formulae for $S$ and $d S / d t$ are developed in [8] for a length $R$ line segment moving parallel with the image plane. On the contrary the simulation includes a $3 \mathrm{D}$ vertex set which is projected to the image plane, this can lead to differences mainly for intruders coming from large $\beta$ and $\alpha$ directions. To prove this assumption simulations were conducted for the case with 28.6\% FA rate applying a rectangle moving parallel with the image plane as intruder. The FA rate decreased to $0 \%$.

TABLE I

Summary OF TEST RESUlTS: PERCENT OF C DECISIONS

\begin{tabular}{|c||c|c|c|c|c|c|}
\hline $\mathrm{R}[\mathrm{m}]$ & $V_{i}[\mathrm{~m} / \mathrm{s}]$ & & & CPA & & \\
\hline & & 0 & 10 & 20 & 60 & 120 \\
\hline \hline & 17 & 100 & 100 & 100 & 0 & 0 \\
\hline 1.2 & 20 & 100 & 100 & 100 & 6 & 0 \\
\hline & 23 & 100 & 100 & 100 & 0 & 0 \\
\hline \hline & 10 & 100 & 100 & 100 & 40 & 0 \\
\hline 5 & 25 & 100 & 100 & 100 & 34 & 3 \\
\hline & 40 & 100 & 100 & 100 & 31 & 11.4 \\
\hline \hline & 37 & 100 & 100 & 100 & 45.7 & 25.7 \\
\hline 10 & 64 & 100 & 100 & 100 & 37.1 & 17.1 \\
\hline & 127 & 100 & 100 & 100 & 34.3 & 17.1 \\
\hline \hline & 52 & 100 & 100 & 100 & 66 & 46 \\
\hline 20 & 145 & 100 & 100 & 100 & 40 & 26 \\
\hline & 256 & 100 & 100 & 100 & 34.3 & 22.9 \\
\hline \hline & 133 & 100 & 100 & 100 & 57.1 & 51.4 \\
\hline 40 & 222 & 100 & 100 & 100 & 48.6 & 34.3 \\
\hline & 265 & 100 & 100 & 100 & 45.7 & 31.4 \\
\hline \hline & 205 & 100 & 100 & 100 & 57 & 40 \\
\hline 60 & 241 & 100 & 100 & 100 & 48.6 & 43 \\
\hline & 257 & 100 & 100 & 100 & 51.4 & 37.1 \\
\hline \hline
\end{tabular}

A possible solution is to increase $\dot{S}_{L I M 0}$ with heuristic tuning until it preserves the detections (no miss detect for small CPA) but decreases FA rate. A better solution can be the ego motion compensation for intruder sizes and the derivation of $S$ and $d S / d t$ formulae for complicated 3D intruder shapes.

Considering the miss detection rate $(0 \%)$ the $70 \%$ required effectiveness is well satisfied. The only future task is to decrease FA rate.

\section{First CAMERA TEST RESUlts}

First test results were obtained with a ground fixed camera towards which a small RC aircraft has flown several raids. An example screenshot from observation to close intruder can be seen in Fig. 8.

Results from a close to collision and a non-collision scenario are shown in Fig. 9, 10 and 11 considering the same time span for the plots. All image processing is done as in the simulations except for ego motion compensation because camera is fixed and so ego motion is zero. The thresholds are also calculated considering $k_{b}$ as in (7). 


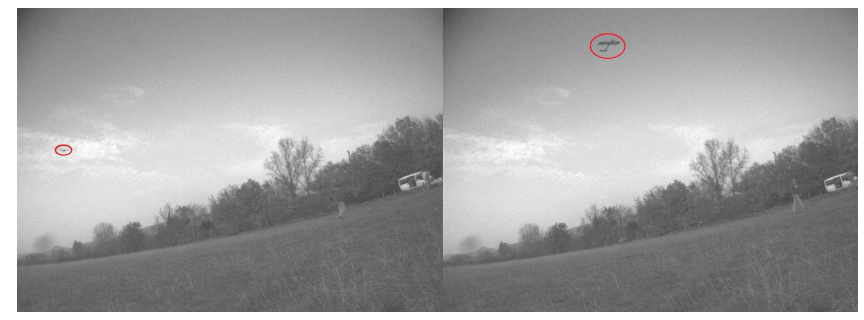

Fig. 8. Screenshots with real camera (left side: observation, right side: close intruder)

Fig. 9 shows that the motion of intruder in image is much smaller for raid 3 than for raid 1 , so raid 3 can be a close to collision situation.

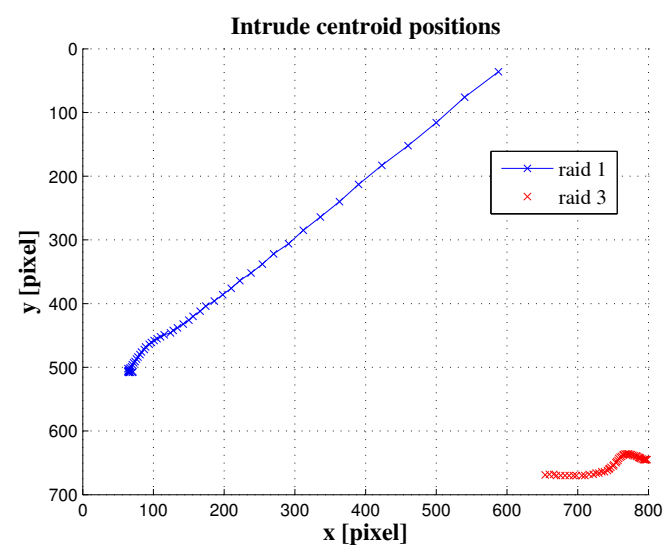

Fig. 9. Intruder positions
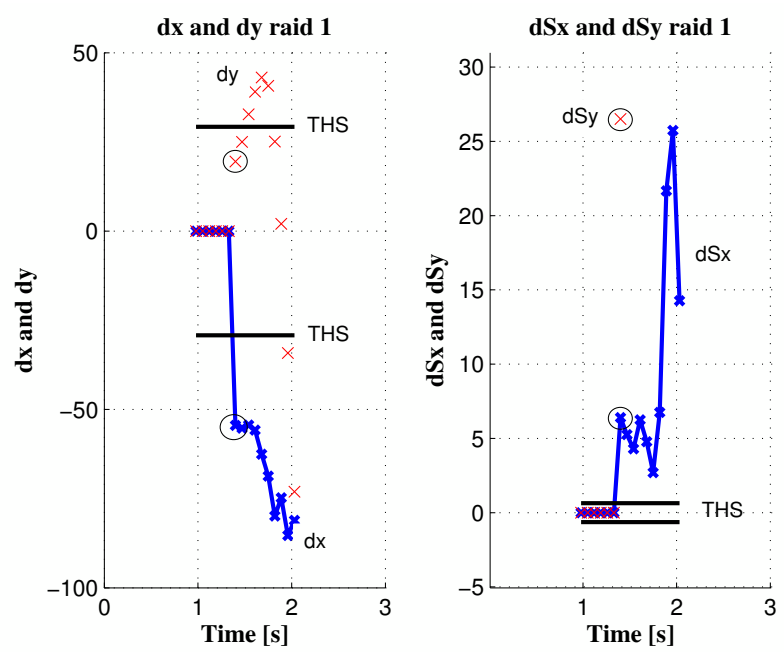

Fig. 10. Decision variables and limits, raid 1
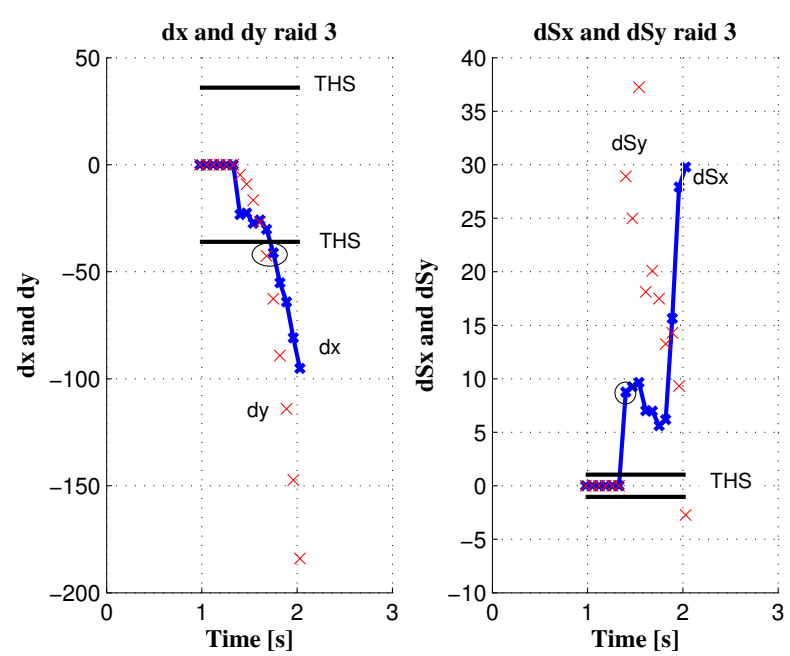

Fig. 11. Decision variables and limits, raid 3

Fig. 10 shows that for raid 1 both $d x=d x / d t, d S x=$ $d S x / d t$ and $d S y=d S y / d t$ exceeds the thresholds at the calculation of first nonzero values (denoted by circles). This case (if all thresholds are violated) the algorithm considers the case as non-collision. In case of raid 3 (Fig. 11) $d S x$ and $d S y$ exceed the threshold immediately and $d y=d y / d t$ exceeds it later (first values out of the thresholds are denoted by circles). This leads to a collision decision. So, the decisions are correct, however larger thresholds would be better to make the decisions later not at the first nonzero derivatives. Possibilities will be examined in the future for larger set of test data.

\section{CONCLUSION}

This paper develops and tests a non-heuristic threshold selection method for image parameter-based aircraft sense and avoid . First, it summarizes the theoretical basis and the proposed steps of threshold selection. Then it characterizes own craft and possible intruder threats considering real aircraft data ranging from GA to large airliner / transport. It derives closed form relations between aircraft characteristic sizes and velocities considering minimum and maximum velocity bounds also. It also selects the appropriate S\&A system effectiveness requirement based-on [10]. After characterizing the possible threats the main properties of the applied SIL simulation environment are listed.

The main part of the paper executes the derived formulae following step by step the proposed threshold selection methodology. It determines the guaranteed collision and noncollision decision ranges considering relative miss distance (CPA).

Results from the simulation campaign are summarized in a table. The miss detection rate of the method is $0 \%$ which is an excellent result. On the contrary the false alarm rate can be as large as $51.4 \%$ which shows that the method is over conservative. The cause of this is the lack of ego motion compensation for intruder image size and the application of more simple formulae for image size change then required. 
First results with real camera images are also presented applying a ground based camera and a small RC aircraft as intruder. The results are promising, near collision and noncollision scenarios can be well distinguished.

The further developments should target the following topics:

- Solve ego motion compensation of intruder image size and make the related formulae more accurate if possible.

- Consider limited camera field of view and also effective field of view (see [14]) in intruder observation and during avoidance maneuver.

- Propose solutions when intruder is non-cooperative but also equipped with S\&A system. Some right hand rule or other should be introduced to avoid crashes if all two aircrafts execute avoidance.

- Detailed camera test with more scenarios and airborne camera.

\section{REFERENCES}

[1] "Roadmap for the integration of civil Remotely-Piloted Aircraft Systems into the European Aviation System," European RPAS Steering Group, Tech. Rep., 2013.

[2] Department of Defense, USA, "Unmanned Aircraft System Airspace Integration Plan," March 2011.

[3] L. R. Salazar, R. Sabatini, S. Ramasamy, and A. Gardi, "A Novel System for Non-Cooperative UAV Sense-And-Avoid," in In Proceedings of European Navigation Conference 2013 (ENC 2013), April 2013.

[4] B. Vanek, T. Peni, A. Zarandy, J. Bokor, T. Zsedrovits, and T. Roska, "Performance Characteristics of a Complete Vision Only Sense and Avoid System," in in Proceedings of AIAA GNC 2012 (Guidance, Navigation and Control Conference), no. AIAA 2012-4703, Minneapolis, Minnesota, August 2012, pp. 1-15.

[5] S. D. B, "Reactive Image-based Collision Avoidance System for Unmanned Aircraft Systems," Master's thesis, Australian Res earch Centre for Aerospace Automation, May 2011.

[6] Y. Watanabe, "Stochastically Optimized Monocular Vision-based Navigation and Guidance," Ph.D. dissertation, Georgia Institute of Technology, 2008.

[7] E. W. Frew, "Observer Trajectory Generation for Target-Motion Estimation Using Monocular Vision," Ph.D. dissertation, Stanford University, 2003.

[8] R. J. Bootsma, "Predictive Information and the Control of Action: What You See is What You Get," Int. J. Sport Psychol, vol. 22, pp. 271-278, 1991.

[9] R. W. Beard and T. W. McLain, Small Unmanned Aircraft: Theory and Practice. Princeton University Press, 2012.

[10] R. Melnyk, D. Schrage, V. Volovoi, and H. Jimenez, "Sense and Avoid Requirements for Unmanned Aircraft Systems Using a Target Level of Safety Approach," Risk Analysis, vol. 34, no. 10, pp. 1894-1906, 2014.

[11] [Online]. Available: http://www.airliners.net/aircraft-data/

[12] A. Zarandy, Z. Nagy, B. Vanek, T. Zsedrovits, A. Kiss, and M. Nemeth, "A Five-Camera Vision System for UAV Visual Attitude Calculation and Collision Warning," in Computer Vision Systems, Lecture Notes in Computer Science, Saint Petersburg, Russia, July 2013, pp. 11-20.

[13] D. P. Jack, K. D. Hoffler, and S. C. Johnson, "Evaluation of the Trade Space Between UAS Maneuver Performance and SAA System Performance Requirements," NASA Langley Research Center, Tech. Rep., 2014.

[14] P. Bauer, B. Vanek, T. Peni, T. Zsedrovits, B. Pencz, A. Zarandy, and J. Bokor, "Aircraft Trajectory Tracking with Large Sideslip Angles for Sense and Avoid Intruder State Estimation," in Proc. of 22nd Mediterranean Conference on Control \& Automation (MED'14). Palermo, Italy: IEEE, June 2014, pp. 1287-1292. 